\title{
Manifestation of classical wave delays in a fully-quantized model of the scattering of a single photon
}

\author{
Thomas Purdy $\dagger \S$, Daniel R Taylor $\ddagger \|$ and Martin Ligare $\uparrow$ \\ $\dagger$ Department of Physics, Carnegie Mellon University, Pittsburgh, PA 15213, \\ USA \\ $\ddagger$ Department of Physics, Bucknell University, Lewisburg, PA 17837, USA
}

\begin{abstract}
We consider a fully-quantized model of spontaneous emission, scattering, and absorption, and study propagation of a single photon from an emitting atom to a detector atom both with and without an intervening scatterer. We find an exact quantum analog to the classical complex analytic signal describing an electromagnetic wave scattered by a medium of charged oscillators. This quantum signal exhibits classical phase delays. We define a time of detection which, in the appropriate limits, exactly matches the predictions of a classically defined delay for light propagating through a medium of charged oscillators. The fully quantized model provides a simple, unambiguous, and causal interpretation of delays that seemingly imply speeds greater than $c$ in the region of anomalous dispersion.

PACS numbers: 32.80.-t, 42.25.Bs, 42.50.Ct
\end{abstract}

\section{Introduction}

The subtleties of appropriately defining the speed of light pulses in dispersive media have been investigated for over a century. Although a careful classical analysis was performed early in the twentieth century (see, for example, the papers collected in reference [1]), recent observations of anomalously slow [2, 3] and anomalously fast [4, 5] speeds of light have returned studies of light propagation to the pages of contemporary physics journals, and a recent review so-called superluminal propagation is contained in [6. In this paper we study the scattering of single spontaneously emitted photons and develop a simple fully-quantized microscopic model of light propagation in a dielectric medium. We also compare this quantized model to a simple microscopic classical model of scattering from charged oscillators. Although our models are limited to the simplest case of weak scattering in linear dielectrics, they serve as a basis for understanding propagation in more complex media at the quantum level.

Classical pulses can be understood as the superposition of the incident field with fields re-radiated by the atoms of the medium. This point of view has been articulated clearly by Feynman [7, with recent elaborations by Sherwood [8] and Milonni [9]. The re-radiated fields are phase-shifted and attenuated relative to the incident field,

$\S$ Current address: Department of Physics, University of California, Berkeley, CA 94720-7300, USA

|| Current address: Department of Physics, Boston University, Boston, MA 02215, USA

I To whom correspondence should be addressed (mligare@bucknell.edu) 
and the leading edges of all fields propagate at the vacuum speed of light $c$. We identify quantum mechanical quantities that have a striking quantitative parallel in the complex analytic signal describing fields in the classical model. Although the state vector describing the system has no absolute overall phase, the scattering-induced phase shift of the classical field is manifested in the description of the quantum field as well as in the probability amplitude for atoms subsequently excited by the field. The pulse re-shaping of classical fields that leads to group delays is also evident in our quantum model, and results in delays that are equivalent in the classical and quantum models. All delays are clearly due to the superposition of quantum effects that propagate at the vacuum speed of light $c$, in spite of the appearance of delays that seemingly imply so-called superluminal velocities. The parallels between classical and quantum scattered fields facilitate the understanding of delays of single photons in terms of classical concepts.

The classical medium we consider consists of charged point particles attached to their equilibrium positions by linear restoring forces. This model is considered in undergraduate textbooks 7 10. The classical medium has an index of refraction which for a dilute collection of scatterers depends linearly on the density of the scatterers, and the index of refraction determines the phase and group velocities of radiation in the medium.

Our quantum system consists of a set of two-level atoms at fixed positions interacting with the quantized modes of a one-dimensional multimode optical cavity. An initially excited atom spontaneously emits radiation into the modes of the cavity (a photon); the radiation is scattered by a second atom; and a third atom serves as a detector. We find an analytical expression describing the excitation of the detector atom, and an expression for the expectation value of the space- and time-dependent electromagnetic field intensity. We then identify an exact quantum analog to the complex analytic signal of a classical field. The scattering-induced time delays in the quantum mechanical quantities thus have direct parallels in classical delays. We also find a simple, unambiguous, and causal interpretation at the quantum level of a superluminal velocity that occurs in the region of anomalous dispersion very near to an atomic resonance.

We note that the results obtained analytically in this paper may also be observed using straightforward numerical techniques like those used in references [11, 12, 13, 14]. Our work bears a particularly close relationship to the numerical work reported in [15], in which quantum interference effects are observed in the scattering of single-photon wavepackets.

We review the classical model in section 2 and in section 3 we discuss our quantum mechanical model and present our analytical solution. (We leave the details of the derivation to the Appendix.) We highlight the similarity of the quantum and classical dynamics in section 4 and in section 5 we investigate a delay that seemingly implies superluminal propagation in both the classical and quantum models.

\section{Review of Scattering from Classical Oscillators}

A simple classical model of the interaction of radiation with matter consists of an electromagnetic plane wave driving electrons attached to molecules by linear springs [7. 10. This simple model is able to account for the attenuation and phase shift of waves transmitted through a dilute material comprised of such oscillators.

For a plane wave normally incident on a dielectric slab with thickness $\Delta z$ and 
real index of refraction $n$, the delay in the arrival of a point of constant phase on the far side of the medium (compared to a wave traveling in vacuum) is determined by the phase velocity $v_{\phi}=\omega / k=c / n$. This delay is given by

$$
\Delta t_{\phi}=\frac{\Delta z}{v_{\phi}}-\frac{\Delta z}{c}=\frac{\Delta z}{c}(n-1) .
$$

The delay in the arrival of the peak of a modulation envelope of a quasi-monochromatic pulse is determined by the group velocity $v_{\mathrm{g}}=\mathrm{d} \omega / \mathrm{d} k=c /\left(n+\omega \frac{\mathrm{d} n}{\mathrm{~d} \omega}\right)$, and is given by

$$
\Delta t_{\mathrm{g}}=\frac{\Delta z}{v_{\mathrm{g}}}-\frac{\Delta z}{c}=\frac{\Delta z}{c}\left(n-1+\omega \frac{\mathrm{d} n}{\mathrm{~d} \omega}\right) .
$$

For pulses that are not sufficiently monochromatic, the simple concepts of phase and group velocity are inadequate to characterize all of the effects of pulse-reshaping as the field propagates. Several other velocities and delays have been developed (see, for example, references [16, 17]) and in this paper we focus on a delay determined by the "temporal center of gravity" of the field intensity of a pulse at a fixed position $z$ "downstream" from the dielectric, i.e.,

$$
\Delta t_{\mathcal{E}^{2}} \equiv\left(\frac{\int t \mathcal{E}(z, t)^{2} \mathrm{~d} t}{\int \mathcal{E}(z, t)^{2} \mathrm{~d} t}\right)_{\text {with dielectric }}-\left(\frac{\int t \mathcal{E}(z, t)^{2} \mathrm{~d} t}{\int \mathcal{E}(z, t)^{2} \mathrm{~d} t}\right)_{\text {no dielectric }}
$$

This is closely related to concepts used to define the centrovelocity in [16. For quasimonochromatic pulses far from resonance this delay is equivalent to the group delay, but in general it is necessary to calculate explicitly the field $\mathcal{E}$ in order to determine $\Delta t_{\mathcal{E}^{2}}$. For the spontaneously emitted pulses with Lorentzian spectrums that are considered in this paper we will show that the "temporal-center-of-gravity" delay happens to be equal to twice the group delay.

In order to find classical expressions for the index of refraction and absorption we follow the development outlined by Feynman [7. We consider a monochromatic linearly-polarized plane wave of frequency $\omega$ normally incident on an infinite thin slab of material with density of oscillators $N$ and thickness $\Delta z$. The plane of the slab is normal to the $z$ direction, and located at $z=0$. The oscillators have natural frequency $\omega_{0}$, mass $m$, instantaneous speed $v$, and are assumed to experience a damping force proportional to the first derivative of acceleration, which for sinusoidal oscillations is equivalent to a damping proportional to $-v$. The field on the far side of the slab is the superposition of the incident field and the field scattered by the material. In the steady state the scattered field is proportional to the incident field, with an amplitude and phase shift given by simple resonance theory. The infinite slab geometry considered here creates an effectively one-dimensional model that matches the simple quantum mechanical system we introduce in the following section.

If the incident field is $E_{\mathrm{i}}=E_{0} \exp [-\mathrm{i} \omega(t-z / c)]$, then the scattered field is [7] 10]

$$
\begin{aligned}
E_{\mathrm{s}} & =-\frac{q N \Delta z}{2 \epsilon_{0} c} v \\
& =\mathrm{i} f \frac{\gamma \omega}{\left(\omega_{0}^{2}-\omega^{2}-\mathrm{i} \gamma \omega\right)} E_{\mathrm{i}},
\end{aligned}
$$

where in the last line we have introduced the dimensionless parameter $f=$ $q^{2} N \Delta z /\left(2 m \epsilon_{0} c \gamma\right)$ which characterizes the magnitude of the scattering.. The total transmitted field $E_{\mathrm{t}}$ on the far side of the material is

$$
\begin{aligned}
E_{\mathrm{t}} & =E_{\mathrm{i}}+E_{\mathrm{s}} \\
& =E_{\mathrm{i}}\left[1+\mathrm{i} f\left(\frac{\omega \gamma}{\omega_{0}^{2}-\omega^{2}-\mathrm{i} \gamma \omega}\right)\right] .
\end{aligned}
$$




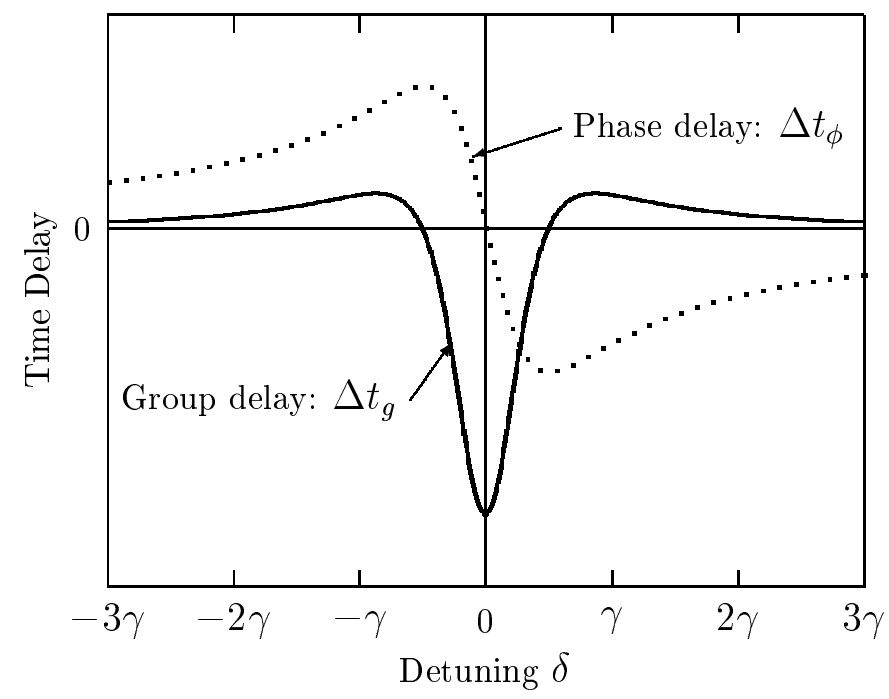

Figure 1. Phase and group delays of a plane wave propagating through a medium of classical oscillators. The group delay is negative in the region of anomalous dispersion, i.e., for $-\gamma / 2<\delta<\gamma / 2$.

In comparing classical predictions with those from a quantum mechanical model we will be interested in the limit in which the detuning from resonance is small compared to the natural frequency of the oscillator, i.e., $\left|\omega-\omega_{0}\right| \ll \omega_{0}$. Taking advantage of this condition we rewrite the transmitted field of equation (5) in terms of the detuning from resonance

$$
\delta=\omega-\omega_{0},
$$

and we approximate other occurrences of $\omega$ with $\omega_{0}$, giving

$$
E_{\mathrm{t}} \simeq E_{\mathrm{i}}\left[1-\mathrm{i} f\left(\frac{\gamma}{2 \delta+\mathrm{i} \gamma}\right)\right] .
$$

For weak scattering, i.e., when $f \ll 1$, the transmitted field is approximately

$$
\begin{aligned}
E_{\mathrm{t}} & \simeq E_{\mathrm{i}}\left[1-f \frac{\gamma^{2}}{\left(4 \delta^{2}+\gamma^{2}\right)}\right]\left[1-\mathrm{i} f \frac{\gamma \delta}{\left(4 \delta^{2}+\gamma^{2}\right)}\right] \\
& \simeq E_{\mathrm{i}} \exp \left[-f \frac{\gamma^{2}}{\left(4 \delta^{2}+\gamma^{2}\right)}\right] \exp \left[-\mathrm{i} f \frac{\gamma \delta}{\left(4 \delta^{2}+\gamma^{2}\right)}\right] .
\end{aligned}
$$

The phase-shift and attenuation given by equation (8) lead to the index of refraction

$$
n=1-\frac{N q^{2}}{m \epsilon_{0} \omega_{0} \gamma} \frac{\gamma \delta}{4 \delta^{2}+\gamma^{2}}
$$

and intensity absorption coefficient

$$
\alpha=\frac{N q^{2}}{m \epsilon_{0} c \gamma} \frac{\gamma^{2}}{\left(4 \delta^{2}+\gamma^{2}\right)} .
$$

Using the expression for the index of refraction given in equation (9), and assuming $\delta \ll \omega_{0}$, the phase and group delays can be written

$$
\Delta t_{\phi} \simeq-\frac{2 f}{\omega_{0}}\left(\frac{\gamma \delta}{4 \delta^{2}+\gamma^{2}}\right)
$$




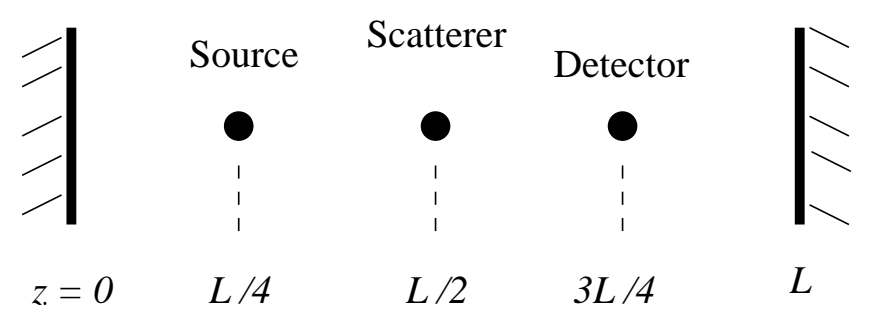

Figure 2. Quantum mechanical model consisting of two-level atoms at fixed positions in a one-dimensional multimode optical cavity.

$$
\Delta t_{\mathrm{g}} \simeq 2 f \gamma \frac{\left(4 \delta^{2}-\gamma^{2}\right)}{\left(4 \delta^{2}+\gamma^{2}\right)^{2}} .
$$

The phase and group delays predicted from the classical model are illustrated in figure 1. The group delay is positive away from resonance, indicating a group velocity less than the vacuum speed of light $c$. In the region of anomalous dispersion near resonance the classical group delay is negative, indicating that the group velocity is greater than $c$. The steep slope of the index of refraction and the accompanying strong and rapidly varying absorption severely distort pulses that are not sufficiently monochromatic, and the standard physical interpretation of $\mathrm{d} \omega / \mathrm{d} k$ as the speed at which the peak of a modulation envelope travels is not valid for such pulses in this region. The "temporal-center-of-gravity" delay retains obvious physical meaning for all pulses, and in section [5] we develop quantum mechanical properties that are analogous to this delay for spontaneously emitted photons. This provides a framework for an unambiguous and causal interpretation of negative values of delays in both classical and quantum models.

Group delays and "temporal-center-of-gravity" delays can be understood as a result of the transient oscillations in the medium [7]. These transient oscillations radiate fields that initially cancel the field of the incident wave, modifying the leading edge of transmitted pulses before they settle down to the steady-state fields given by equation (8). Field transients must be included explicitly in the calculation of delays for pulses in the region of anomalous dispersion. We include a specific calculation of the "temporal-center-of-gravity" delay in section 5

\section{Quantum Model}

The quantum mechanical system we consider is illustrated in figure 2 and consists of three two-level atoms in a one-dimensional multimode optical cavity that extends from $z=0$ to $z=L$. The leftmost atom will initially be in the excited state, and will be the source of the field. The atom in the middle will scatter the radiation emitted by the source atom, and the atom on the right will serve as the detector. The cavity is assumed to be large in the sense that the length $L$ is very much greater than the wavelength of the light emitted by the atoms. (The finite optical cavity does not contribute to the physical phenomena under investigation; it simply provides a convenient quantization volume for the field modes used in our calculations.) In this

section we discuss the standard quantum optical Hamiltonian that we use, and we present the analytical solution for the time dependence of this system. 
The zero-field resonance frequencies of the atoms are labeled $\omega_{j}^{\text {(at) }}$, where $j=1$, 2 , or 3 , and the positions of the atoms will be labeled $z_{j}$. In the results given below we will assume that the atoms are at positions $z_{1}=L / 4, z_{2}=L / 2$, and $z_{3}=3 L / 4$, as illustrated in figure 2 although the delay times we derive do not depend on the exact positions. The standing-wave field modes of the cavity are separated in angular frequency by the fundamental frequency

$$
\Delta_{c}=\pi \frac{c}{L} \text {. }
$$

This mode spacing may be small enough that many modes fall within the natural line-width of the atoms.

For convenience we assume that the frequency of one of the modes corresponds exactly to the resonance frequency of atom 1, the emitting atom, and that the length of the cavity is such that it contains an even number of wavelengths of this mode. We label the frequency of this mode $\omega_{0}=m_{0} \Delta_{c}$, where $m_{0}$ is an integer divisible by 4 . (This assumption affects the details of some of our calculations, but not the existence of an analytic solution, nor any of our results concerning delay times. Classically, this assumption assures that optical path length between any pair of atoms is an integer number of half-wavelengths of the resonant radiation from atom 1.) The other mode frequencies will be enumerated from this mode so that

$$
\omega_{m}=\left(m_{0}+m\right) \Delta_{\mathrm{c}},
$$

where $m=0, \pm 1, \pm 2, \ldots$ Our large-cavity limit also assures that the frequencies of the three atoms are all very much greater than the fundamental frequency of the cavity, i.e., $\omega_{j}^{\text {(at) }} \gg \Delta_{\text {c. }}$. In this limit the atoms interact with a large number of modes, and not simply the resonant mode.

As in the classical case we wish to study the effects of the detuning of the source field on the scattering of the radiation. We use the same symbol $\delta$ as in the classical case to represent the detuning of the field, but in the quantum case the detuning is directly tied to the properties of the source and scattering atoms:

$$
\delta=\omega_{1}^{(\text {at })}-\omega_{2}^{(\text {at })} .
$$

The detector atom (atom 3 ) is assumed to have the same resonance frequency as the source atom, i.e., $\omega_{1}^{(\text {at })}=\omega_{3}^{(\text {at })}$.

We use as basis states the eigenstates of the atomic plus free-field Hamiltonian

$$
\begin{aligned}
\hat{H}_{0} & =\hat{H}_{\text {atoms }}+\hat{H}_{\text {field }} \\
& =\sum_{j=1}^{3} \hbar \omega_{j}^{(\text {at })} \sigma_{j}^{z}+\sum_{m} \hbar \omega_{m} a_{m}^{\dagger} a_{m},
\end{aligned}
$$

where $\sigma_{j}^{z}$ is the third component of the atomic pseudo-spin operator of atom $j$, and $a_{m}$ and $a_{m}^{\dagger}$ are the lowering and raising operators for the $m^{\text {th }}$ field mode. (We have re-zeroed the energy scale to remove zero-point energy of the field modes.) The basis states will be denoted as follows:

- $|e, g, g ; 0\rangle$ - Atom 1 excited, atoms 2 and 3 in ground state; no photons in field,

- $|g, e, g ; 0\rangle$ - Atom 2 excited, atoms 1 and 3 in ground state; no photons in field,

- $|g, g, e ; 0\rangle$ - Atom 3 excited, atoms 1 and 2 in ground state; no photons in field,

- $\left|g, g, g ; 1_{m}\right\rangle$ - All atoms in ground state; one photon in field mode with frequency $\left(m_{0}+m\right) \Delta_{\mathrm{c}}$. 
We use the standard electric-dipole and rotating-wave approximations in the interaction Hamiltonian [18, 19, 20, to give

$$
\begin{aligned}
\hat{H} & =\hat{H}_{\text {atoms }}+\hat{H}_{\text {field }}+\hat{H}_{\text {interaction }} \\
& =\hat{H}_{0}+\sum_{j=1}^{3} \sum_{m} \hbar\left(g_{j m} a_{m} \sigma_{j}^{+}+g_{j m}^{*} a_{m}^{\dagger} \sigma_{j}^{-}\right),
\end{aligned}
$$

where the strength of the coupling of the $j^{\text {th }}$ atom to the $m^{\text {th }}$ mode of the field is characterized by the constant $g_{j m}$, and $\sigma_{j}^{+}$and $\sigma_{j}^{-}$act as raising and lowering operators for atom $j$.

In our large-cavity limit, $\omega_{j}^{\text {(at) }} \gg \Delta_{\mathrm{c}}$, we can make the approximation that all modes that influence the dynamics of the system are near the atomic resonances, and the atom-field coupling constants are given by

$$
g_{j m}=\Omega_{j} \sin \left[\left(m_{0}+m\right) \pi z_{j} / L\right] .
$$

In the previous equation $\Omega_{j}$ is a constant given by

$$
\Omega_{j}=d_{j}\left(\frac{\omega_{j}^{(\mathrm{at})}}{2 \hbar \epsilon_{0} V}\right)^{1 / 2},
$$

where $d_{j}$ is the dipole matrix element between the levels of atom $j$, and $V$ is the effective volume of the cavity.

Initially only the source atom will be excited and no photons will be present in the field, so that

$$
|\psi(0)\rangle=|e, g, g ; 0\rangle,
$$

and we write the general state of the system as the linear combination

$$
|\psi(t)\rangle=c_{1}(t)|e, g, g ; 0\rangle+c_{2}(t)|g, e, g ; 0\rangle+c_{3}(t)|g, g, e ; 0\rangle+\sum_{m} b_{m}(t)\left|g, g, g ; 1_{m}\right\rangle .
$$

The Schrödinger equation yields the following set of coupled differential equations for the coefficients in equation (21):

$$
\begin{aligned}
& \dot{c}_{j}=-\mathrm{i}\left(\omega_{j}^{(\mathrm{at})} c_{j}+\sum_{m} g_{j m} b_{m}\right) \\
& \dot{b}_{m}=-\mathrm{i}\left(\omega_{m} b_{m}+g_{1 m}^{*} c_{1}+g_{2 m}^{*} c_{2}+g_{3 m}^{*} c_{3}\right) .
\end{aligned}
$$

We solve this set of equations with the Laplace transform technique used by Stey and Gibberd 21. Laplace transforms have also been used to solve the Schrödinger equation in similar problems with two interacting atoms in three-dimensions [22, 23]. Because the Laplace transform technique is not new, and because we would like to focus on analogies with the classical model and physical interpretation, we leave the details of our solution to the appendix, and simply quote our results here.

The general features of the solution giving the time-dependent atomic excitation amplitudes are illustrated in figure 3 . The initially excited atom decays exponentially until $t=0.5 \mathrm{~L} / \mathrm{c}$, the time at which scattered and reflected radiation first returns to the atom. The amplitudes to find the other atoms excited are identically zero until radiation first reaches them: the scattering atom first becomes excited at $t=0.25 \mathrm{~L} / \mathrm{c}$ and the detector atom is first excited at $t=0.5 \mathrm{~L} / \mathrm{c}$. The three decay constants which 

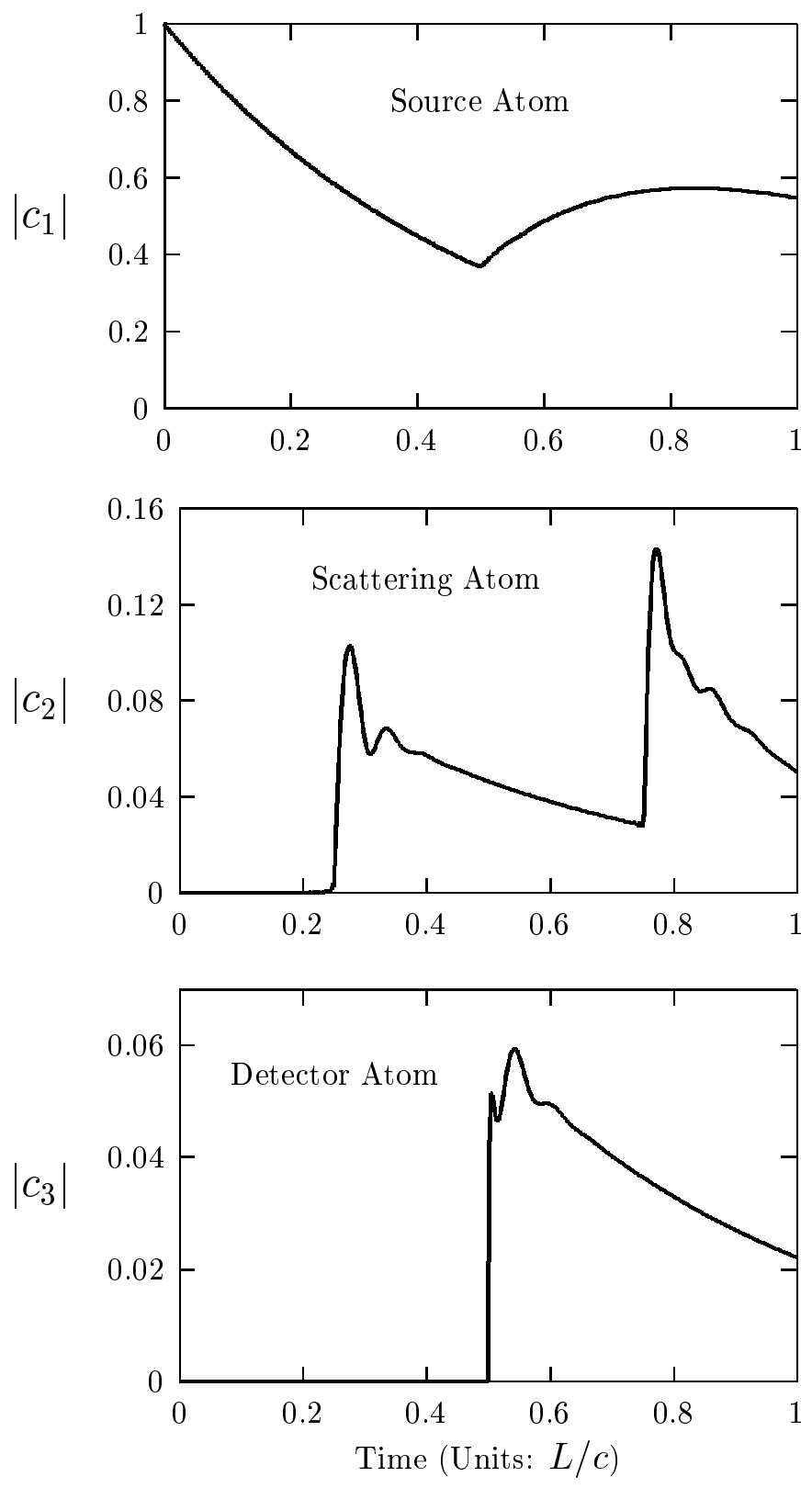

Figure 3. Magnitude of the amplitudes for the atoms to be in the excited state, starting from the state $|\psi(0)\rangle=|e, g, g ; 0\rangle$. The decay rates of the atoms are $\gamma_{1}=4, \gamma_{2}=64$, and $\gamma_{3}=1024$ (in the units of the figure) and the detuning is $\delta=1.56 \gamma_{2}$. 
characterize the spontaneous emission rates of each of the atoms emerge naturally in terms of the parameters of the Hamiltonian as

$$
\gamma_{j}=\frac{\pi\left|\Omega_{j}\right|^{2}}{\Delta_{c}}=\left|\Omega_{j}\right|^{2} \frac{L}{c} .
$$

The causal nature of the dynamics is evident in that all disturbances are propagated at the speed of light $c$ via the quantum field. The abrupt changes in the complex amplitudes at intervals of $0.5 \mathrm{~L} / \mathrm{c}$ are a manifestation of the finite speed of light and the atomic spacing of $0.25 \mathrm{~L}$.

The abrupt changes are manifested in our analytic solution for the complex amplitudes $c_{j}(t)$ and $b_{m}(t)$ as sums of terms with step functions that "turn on" at successively later intervals of $0.5 \mathrm{~L} / \mathrm{c}$. In the following formulas giving these amplitudes we truncate the expressions so that only the first excitations of atoms 2 and 3 are included. We also note that the following equations are specific in some details to the atomic positions $z_{i}$ used in this paper. The positions of the atoms enter via the coupling constants $g_{j m}$, and changes in positions will result in changes in "turnon" times and relative phases of terms. One manifestation of our large-cavity limit $\omega \gg \Delta_{c}$ is that the source atom decay at the free-space rates is clearly visible before interruption by reflected and scattered radiation, for example at times $t<0.5 \mathrm{~L} / \mathrm{c}$ in figure 3 The initial atomic decay rates given by equation (24) are not affected by the positions of the atoms; the effects of position dependence in the coupling constants compensate to keep the total initial decay rates independent of position. (Examples of the relationship between interrupted free-space decay and cavity-modified decay rates are given in 24.) Later in this paper we will consider decay times that are much shorter than $L / c$ so that the effects of all initial excitations are complete before reflected or mutiply scattered radition excites the atoms. The conclusions of this paper concerning delay times are unaffected by any details of position dependence.

The complete time dependence of the system is given by the following set of amplitudes (in which the time is scaled in units of $L / c$ ):

$$
\begin{aligned}
& \begin{array}{c}
c_{1}(t)=\exp \left(-\frac{\gamma_{1}}{2} t\right)+\Theta\left(t-\frac{1}{2}\right) \frac{\gamma_{1}}{\left(\gamma_{1}-\gamma_{2}+\mathrm{i} 2 \delta\right)^{2}} \\
\times\left\{\exp \left[-\frac{\gamma_{1}}{2}\left(t-\frac{1}{2}\right)\right]\left[\frac{\left(\gamma_{1}-2 \gamma_{2}+\mathrm{i} 2 \delta\right)\left(\gamma_{1}-\gamma_{2}+\mathrm{i} 2 \delta\right)}{2}\left(t-\frac{1}{2}\right)-\gamma_{2}\right]\right. \\
\left.\quad+\gamma_{2} \exp \left[-\left(\frac{\gamma_{2}}{2}-\mathrm{i} \delta\right)\left(t-\frac{1}{2}\right)\right]\right\}+\cdots \\
c_{2}(t)=\Theta\left(t-\frac{1}{4}\right) \frac{\sqrt{\gamma_{1} \gamma_{2}}}{\gamma_{1}-\gamma_{2}+\mathrm{i} 2 \delta}\left\{\exp \left[-\frac{\gamma_{1}}{2}\left(t-\frac{1}{4}\right)\right]-\exp \left[-\left(\frac{\gamma_{2}}{2}-\mathrm{i} \delta\right)\left(t-\frac{1}{4}\right)\right]\right\}+\cdots \\
c_{3}(t)=\Theta\left(t-\frac{1}{2}\right) \sqrt{\gamma_{1} \gamma_{3}}\left\{\exp \left[-\frac{\gamma_{1}}{2}\left(t-\frac{1}{2}\right)\right] \frac{\left(\gamma_{1}+\mathrm{i} 2 \delta\right)}{\left(\gamma_{1}-\gamma_{3}\right)\left(\gamma_{1}-\gamma_{2}+\mathrm{i} 2 \delta\right)}\right. \\
\quad-\exp \left[-\left(\frac{\gamma_{2}}{2}-\mathrm{i} \delta\right)\left(t-\frac{1}{2}\right)\right] \frac{\gamma_{2}}{\left(\gamma_{1}-\gamma_{2}+\mathrm{i} 2 \delta\right)\left(-\gamma_{2}+\gamma_{3}+\mathrm{i} 2 \delta\right)} \\
\left.\quad+\exp \left[-\frac{\gamma_{3}}{2}\left(t-\frac{1}{2}\right)\right] \frac{\left(\gamma_{3}+\mathrm{i} 2 \delta\right)}{\left(-\gamma_{1}+\gamma_{3}\right)\left(-\gamma_{2}+\gamma_{3}+\mathrm{i} 2 \delta\right)}\right\}+\cdots \\
b_{m}(t)=\frac{\mathrm{i} 2 g_{1 m}}{\left(\gamma_{1}-\mathrm{i} 2 m \pi\right)}\left[\exp \left(-\frac{\gamma_{1}}{2} t\right)-\exp (-\mathrm{i} m \pi t)\right]+\Theta\left(t-\frac{1}{4}\right) \mathrm{i} 2 g_{2 m} \sqrt{\gamma_{1} \gamma_{2}}
\end{array}
\end{aligned}
$$




$$
\begin{aligned}
& \times\left\{\frac{\exp \left[-\frac{\gamma_{1}}{2}\left(t-\frac{1}{4}\right)\right]}{\left(\gamma_{1}-\mathrm{i} 2 m \pi\right)\left(\gamma_{1}-\gamma_{2}+\mathrm{i} 2 \delta\right)}+\frac{\exp \left[-\mathrm{i} m \pi\left(t-\frac{1}{4}\right)\right]}{\left(\gamma_{1}-\mathrm{i} 2 m \pi\right)\left[\gamma_{2}-\mathrm{i} 2(\delta+m \pi)\right]}-\right. \\
& \left.-\frac{\exp \left[-\left(\frac{\gamma_{2}}{2}+\mathrm{i} \delta\right)\left(t-\frac{1}{4}\right)\right]}{\left[\gamma_{2}-\mathrm{i} 2(\delta+m \pi)\right]\left(\gamma_{1}-\gamma_{2}+\mathrm{i} 2 \delta\right)}\right\}+\cdots
\end{aligned}
$$

(We note that if we had made a different assumption about the size of resonant wavelength relative to the length of the cavity in our discussion immediately preceding equation (14), then the complex phases associated with terms in the equations above would be different.)

In the following sections we will focus on two quantities: $c_{3}(t)$, the amplitude to find the detector atom excited, and $\left\langle\hat{\mathcal{E}}^{2}\right\rangle$ the expectation value of the square of the electric field operator, which is proportional to the field intensity. (The expectation value of the field operator itself is zero for any state with the form of equation (21).) In our investigation of $c_{3}(t)$ we will consider only the displayed term in equation (27) describing the initial excitation of the detector atom. Similarly, we will investigate $\left\langle\hat{\mathcal{E}}^{2}\right\rangle$ in regions to the right of the scattering atom, and at times that exclude multiple scattering effects.

It is useful to rewrite $c_{3}(t)$ as the sum of two pieces: the amplitude $c_{3}^{0}(t)$ for atom 3 to be excited in the absence of the scattering atom (or, equivalently, when $\gamma_{2}=0$ ), and $c_{3}^{\mathrm{s}}(t)$, the amplitude that is attributable to scattering. The total amplitude is thus

$$
c_{3}(t) \equiv c_{3}^{0}(t)+c_{3}^{\mathrm{s}}(t) .
$$

Setting $\gamma_{2}=0$ in equation (27) gives

$c_{3}^{0}(t)=\Theta\left(t-\frac{1}{2}\right) \frac{\sqrt{\gamma_{1} \gamma_{3}}}{\gamma_{1}-\gamma_{3}}\left\{\exp \left[-\frac{\gamma_{1}}{2}\left(t-\frac{1}{2}\right)\right]-\exp \left[-\frac{\gamma_{3}}{2}\left(t-\frac{1}{2}\right)\right]\right\}$,

and subtracting this from equation (27) gives

$$
\begin{aligned}
c_{3}^{\mathrm{s}}(t)=\Theta(t & \left.-\frac{1}{2}\right) \gamma_{2} \sqrt{\gamma_{1} \gamma_{3}}\left\{\frac{\exp \left[-\frac{\gamma_{1}}{2}\left(t-\frac{1}{2}\right)\right]}{\left(\gamma_{1}-\gamma_{3}\right)\left(\gamma_{1}-\gamma_{2}+\mathrm{i} 2 \delta\right)}+\frac{\exp \left[-\left(\frac{\gamma_{2}}{2}-\mathrm{i} \delta\right)\left(t-\frac{1}{2}\right)\right]}{\left(\gamma_{1}-\gamma_{2}+\mathrm{i} 2 \delta\right)\left(-\gamma_{2}+\gamma_{3}+\mathrm{i} 2 \delta\right)}\right. \\
& \left.+\frac{\exp \left[-\frac{\gamma_{3}}{2}\left(t-\frac{1}{2}\right)\right]}{\left(-\gamma_{1}+\gamma_{3}\right)\left(-\gamma_{2}+\gamma_{3}+\mathrm{i} 2 \delta\right)}\right\} .
\end{aligned}
$$

As an alternative to finding the time-dependence of the excitation amplitude for the detector atom, we can characterize the transmitted field itself without recourse to the details of the detector. Standard photodetection theory 25] suggests calculation of the expectation value $\left\langle\hat{\mathcal{E}}^{(-)}(z, t) \hat{\mathcal{E}}^{(+)}(z, t)\right\rangle$, where $\hat{\mathcal{E}}^{(+)}(z, t)$ and $\hat{\mathcal{E}}^{(-)}(z, t)$ correspond to the decomposition of the interaction representation field operator into positive and negative frequency parts. This is equivalent to the calculation of the expectation value of the normally ordered intensity operator $\left\langle: \hat{\mathcal{E}}^{2}:\right\rangle$.

Using the electric field operator in the form given in reference [18, we write the expectation value of the square of the field as

$$
\left\langle: \hat{\mathcal{E}}^{2}:\right\rangle=\left\langle\psi(t)\left|:\left\{\sum_{m}\left(\frac{\hbar \omega_{m}}{\epsilon_{0} V}\right)^{1 / 2}\left(a_{m}+a_{m}^{\dagger}\right) \sin \left[\left(m_{0}+m\right) \frac{\pi z}{L}\right]\right\}^{2}:\right| \psi(t)\right\rangle .
$$

In the limit considered in this paper we can replace the frequencies $\omega_{m}$ under the radical with the constant $\omega_{1}^{(\text {at) }}$. After expanding the state vector as in equation (21), 
normally ordering the operators, and evaluating the sums, the expectation value can be written in terms of the amplitudes $b_{m}(t)$ to find the photon in the various cavity modes:

$$
\left\langle: \hat{\mathcal{E}}^{2}:\right\rangle=\left(\frac{2 \hbar \omega_{1}^{(\mathrm{at})}}{\epsilon_{0} V}\right)\left|\sum_{m} b_{m}(t) \sin \left[\left(m_{0}+m\right) \frac{\pi z}{L}\right]\right|^{2} .
$$

Evaluation of this expression gives a space- and time-dependent representation of the localization of the energy of the photon 13 14.

The expression for $\left\langle: \hat{\mathcal{E}}^{2}:\right\rangle$ in equation (33) is the square of a complex number that is analogous to the classical complex analytic signal. We label this quantity $\mathcal{E}_{\text {q.m }}$, i.e.,

$$
\mathcal{E}_{\text {q.m. }}=\left(\frac{2 \hbar \omega_{1}^{(\mathrm{at})}}{\epsilon_{0} V}\right)^{1 / 2} \sum_{m} b_{m}(t) \sin \left[\left(m_{0}+m\right) \frac{\pi z}{L}\right] .
$$

The overall phase of this quantity is clearly arbitrary; in what follows we retain the phase that comes from a direct evaluation this equation.

We note that quantity $\mathcal{E}_{\text {q.m. }}$. is very closely related to what has been identified as "the 'electric field' associated with [a] single photon state" by Scully and Zubairy [25]. They consider states which are the product of separable atomic and field states. They conclude that for single photon states $\left|\psi_{\gamma}\right\rangle$ the quantity $\left\langle 0\left|\hat{E}^{(+)}\right| \psi_{\gamma}\right\rangle$ "can be interpreted as a kind of a wave function for a photon." They also demonstrate the classical nature of this quantity for field states produced by spontaneous emission. The present paper extends this kind of analysis to define an analog to the classical analytic signal even in cases in which field and atomic variables are entangled, and it extends the quantum-classical correspondence to fields that include scattering from two-level atoms.

For ease of comparison with previous results for the detector atom, we fix $z=3 L / 4$ in what follows. With no scattering atom present we find (see Appendix)

$$
\mathcal{E}_{\text {q.m. }}^{0}=-\mathrm{i} \Theta\left(t-\frac{1}{2}\right)\left(\frac{\hbar \omega_{1}^{(\mathrm{at})} \gamma_{1} \pi}{2 \epsilon_{0} V \Delta_{\mathrm{c}}}\right)^{1 / 2} \exp \left[-\frac{\gamma_{1}}{2}\left(t-\frac{1}{2}\right)\right] .
$$

The energy density passing the point $z=3 L / 4$ exhibits an abrupt turn-on (because of the initial conditions we have chosen) followed by exponential decay [13, 14, 25]. With a scattering atom present at $z=L / 2$ we find

$$
\begin{aligned}
\mathcal{E}_{\text {q.m. }}=-\mathrm{i} \Theta(t & \left.-\frac{1}{2}\right)\left(\frac{\hbar \omega_{1}^{(\text {at })} \gamma_{1} \pi}{2 \epsilon_{0} V \Delta_{\mathrm{c}}}\right)^{1 / 2}\left(\exp \left[-\frac{\gamma_{1}}{2}\left(t-\frac{1}{2}\right)\right]-\mathrm{i} \frac{\gamma_{2}}{\left[2 \delta-\mathrm{i}\left(\gamma_{1}-\gamma_{2}\right)\right]}\right. \\
& \left.\times\left\{\exp \left[-\frac{\gamma_{1}}{2}\left(t-\frac{1}{2}\right)\right]-\exp \left[-\left(\frac{\gamma_{2}}{2}-\mathrm{i} \delta\right)\left(t-\frac{1}{2}\right)\right]\right\}\right) .
\end{aligned}
$$

Using only the first exponential term leads to the previous result with no scattering atom present; the effect of the scattering is contained in the remaining terms. Calculation of the expectation value of the square of the field will exhibit interference between the terms of this expression, leading to effects similar to those noted in reference [15]. 


\section{Comparison of classical and quantum mechanical scattering}

In order to compare the quantum mechanical "pulses" derived in section 3 to classical analogs it is necessary to extend the treatment reviewed in section 2 to include the effects of transients. This can be done in the frequency domain using Fourier techniques, or in the time domain by explicitly including transients in the solution of the equation of motion of the charged oscillators, and calculating the fields re-radiated by the transients. The quantum mechanical results of section 3 have been derived in the time domain, and to emphasize the analogy between the classical and quantum cases we review the classical time domain calculation.

The quantum pulses of equation (35) that are incident on the scattering atom have the classical analog

$$
\mathcal{E}_{\text {cl. }}^{0}=\Theta\left(t-\frac{1}{2}\right) C \exp \left[-\left(\frac{\gamma_{1}}{2}+\mathrm{i} \omega_{1}\right)\left(t-\frac{1}{2}\right)\right],
$$

where $C$ is a constant. Using the field of equation (37) as the driving field in the equation of motion of a driven charged oscillator with damping constant $\gamma_{2}$ and resonance frequency $\omega_{2}$, it is straightforward to find the motion of the oscillator. For the initial conditions $x(0)=0$ and $\dot{x}(0)=0$, and assuming that $\omega_{2} \gg \gamma_{2}$, we have

$$
\begin{aligned}
x(t) \simeq \Theta(t- & \left.\frac{1}{2}\right) \frac{q C / m}{\left(\omega_{2}^{2}-\omega_{1}^{2}+\mathrm{i} \omega_{1}\left(\gamma_{1}-\gamma_{2}\right)+\gamma_{1}^{2} / 4-\gamma_{1} \gamma_{2} / 2\right)} \\
& \times\left\{\exp \left[-\left(\frac{\gamma_{1}}{2}+\mathrm{i} \omega_{1}\right)\left(t-\frac{1}{2}\right)\right]-\exp \left[-\left(\frac{\gamma_{2}}{2}+\mathrm{i} \omega_{2}\right)\left(t-\frac{1}{2}\right)\right]\right\}
\end{aligned}
$$

Calculating the re-radiated field from the motion of the charges as in equation (4), and making the approximations $\delta=\omega_{1}-\omega_{2} \ll \omega_{j}$ and $\gamma_{j} \ll \omega$, gives the scattered field as

$$
\begin{aligned}
\mathcal{E}_{\text {cl. }}^{s}=-\mathrm{i} \Theta(t & \left.-\frac{1}{2}\right) \exp \left(-\mathrm{i} \omega_{1} t\right) \frac{C f \gamma_{2}}{\left[2 \delta-\mathrm{i}\left(\gamma_{1}-\gamma_{2}\right)\right]} \\
& \times\left\{\exp \left[-\frac{\gamma_{1}}{2}\left(t-\frac{1}{2}\right)\right]-\exp \left[-\left(\frac{\gamma_{2}}{2}-\mathrm{i} \delta\right)\left(t-\frac{1}{2}\right)\right]\right\} .
\end{aligned}
$$

We note that the functional form of this equation giving the classical scattered field is identical to the portion of equation (36) describing the quantum scattered field. In addition, we note that this functional form is also reflected in $c_{3}(t)$. In the limit of rapid detector response, i.e., $\gamma_{3} \gg \gamma_{1}, \gamma_{2}$, this amplitude is

$$
\begin{aligned}
c_{3}(t) \simeq-\Theta(t & \left.-\frac{1}{2}\right) \sqrt{\frac{\gamma_{1}}{\gamma_{3}}}\left(\exp \left[-\frac{\gamma_{1}}{2}\left(t-\frac{1}{2}\right)\right]-\mathrm{i} \frac{\gamma_{2}}{\left[2 \delta-\mathrm{i}\left(\gamma_{1}-\gamma_{2}\right)\right]}\right. \\
& \left.\times\left\{\exp \left[-\frac{\gamma_{1}}{2}\left(t-\frac{1}{2}\right)\right]-\exp \left[-\left(\frac{\gamma_{2}}{2}-\mathrm{i} \delta\right)\left(t-\frac{1}{2}\right)\right]\right\}\right),
\end{aligned}
$$

and the contribution due to scattering is again the same. (Our use of a "detector" which is an atom identical to the "source," except with a more rapid decay rate, is not meant to correspond to any real experiment. It is an idealization meant to illustrate the effects of the quantum field on a simple system with a fast response.)

Thus we see the exact functional form of the classical field reflected in two quantum mechanical quantities: the probability amplitude of the detector atom $c_{3}(t)$, and in our quantum field "amplitude" $\mathcal{E}_{\text {q.m. }}$. The classical and quantum expressions all include a "steady-state" term (which drops off at the slow decay rate of source atoms, 
$\left.\gamma_{1} / 2\right)$. In the limit $\gamma_{1} \ll \gamma_{2}$ the radiation emitted by the source has a very narrow line-width compared to the scattering atom, and this steady-state term simplifies to the form of equation (7) given by the simple classical theory of section 2

The expression for the quantum field "amplitude" $\mathcal{E}_{\text {q.m. }}$ contains a time-dependent phase that is identical to the phase of the classical field, and the probability amplitude $c_{3}(t)$ contains terms due to incident and scattered fields with relative phases that match classical expectations. The concept of the phase of a quantum field has been the subject of investigation from the early days of quantum mechanics [26] 27]. We note, however, that most previous work on the phase of a quantum field has focused on defining a meaningful and mathematically well-behaved phase operator for linear combinations of multiply occupied states of a single-mode field; in this paper we see manifestations of classical phases in a multimode field which contains a single photon.

In the case of classical scattering considered in section 2 the effect of a single scattering event is considered to be small, and exponential phase rotation and attenuation of the transmitted field are the result of many scattering events. In our quantum model the magnitude of the scattering is determined by $\Omega_{2}$ (or equivalently $\gamma_{2}$ ) which characterizes the coupling of atom 2 to the field. In our one-dimensional model the coupling to the incident field and the decay rate of atom 2 are both completely determined by the single parameter $\Omega_{2}$, which means that it is not possible to make the effect of the scattering small without simultaneously making the linewidth of atom 2 very narrow. In a fully three-dimensional model the decay rate of atom 2 would be the result of the atom's coupling to many more modes, and not just those containing the incident field. This scattering into other modes would reduce the scattering in the forward direction (the direction of the detector) from the amount predicted in our simple model. Of course in a fully-three dimensional model there are other effects also: the fraction of source radiation emitted in the direction of the scatterer (and detector) would be reduced, and the scatterer would be driven by a weaker field and would emit less total radiation. The net effect is that the excitation of the detector due to the scattered field will be reduced relative to the direct excitation. If the relative effect of forward scattering is reduced by a factor $f$, then a more realistic expression for the excitation of the detector atom is

$$
c_{3}(t)=\mathcal{N}\left(c_{3}^{0}(t)+f c_{3}^{\mathrm{s}}(t)\right),
$$

where $\mathcal{N}$ gives an overall reduction in the excitation. We have used the same symbol $f$ here that we used earlier for the dimensionless parameter which characterizes the magnitude of classical scattering in section 2 The quantum superposition of equation (41) is analogous to the classical field superposition of equations (5) and (7).

\section{Temporal-center-of-gravity delay}

The group delay of a classical pulse has a clear interpretation for quasi-monochromatic pulses: it is the delay in the arrival of the peak of a pulse compared to the time expected for propagation through a vacuum. The pulses investigated in this paper have sharp leading edges, and this lack of a smooth modulation envelope means that the results of simple classical theory for quasi-monochromatic pulses should not be expected to be a sufficient guide to full understanding. In this section we investigate "temporalcenter-of-gravity" delays in several classical and quantum mechanical quantities.

The first delay we investigate is derived from $c_{3}(t)$, the amplitude for the detector atom to be excited. As we have argued previously, this amplitude will reflect the 

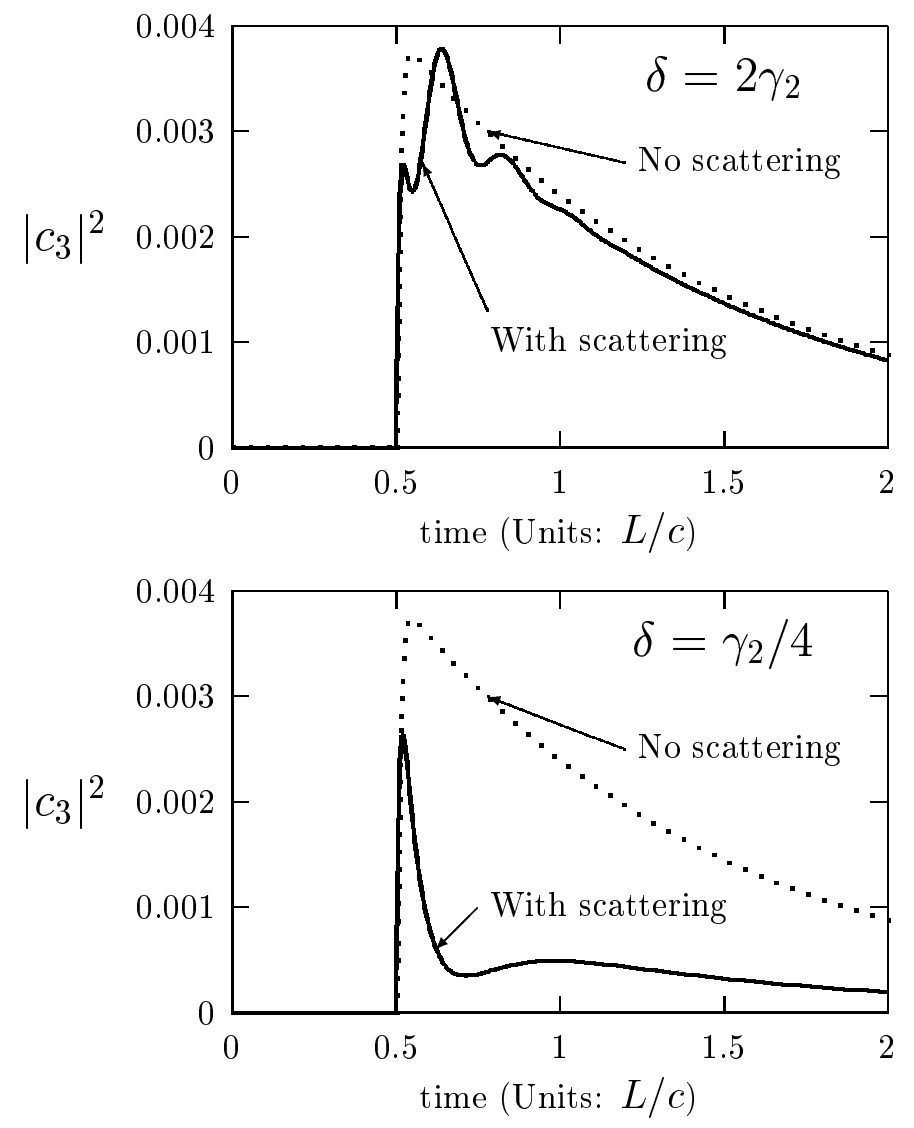

Figure 4. Probability for the detector atom to be excited as a function of time. The top figure is for a detuning $\delta$ in the region of normal dispersion, and the bottom is in the region of anomalous dispersion. The effect of the "pulse re-shaping" is different in the two graphs. In the top graph probability is preferentially removed from earlier times, while the converse is true in the lower graph. The decay rates of the atoms are $\gamma_{1}=1, \gamma_{2}=16$, and $\gamma_{3}=256$ (in the units of the figure).

strength of the incident field in the limit that the response time of this atom is very small compared with other time scales, i.e., $\gamma_{3} \gg \gamma_{1}, \gamma_{2}$. The effect of the scattering on this amplitude is evident in figure 4 in which $\left|c_{3}(t)\right|^{2}$ is plotted for two values of the detuning $\delta$, and also for the case in which no scattering atom is present. (For clarity in this figure we have only included the terms describing the initial "turn-on" of excitation; we have not included effects due to reflection and multiple scattering.) For all detunings, $c_{3}(t)=0$ for all times earlier than $t=0.5 \mathrm{~L} / \mathrm{c}$, as is expected; all effects on the detector atom occur at times that preserve causality. The qualitative shapes of the detector response depend critically on the detuning $\delta$. For detunings in the region of normal dispersion the response is relatively reduced at early times compared to later times, resulting in a qualitative delay in detection of the photon. For detunings in the region of anomalous dispersion $\left(-\gamma_{2} / 2<\delta<\gamma_{2} / 2\right)$ the converse is true. 
We quantify these ideas by identifying an effective arrival time of the photon with the temporal center of gravity of the probability that the detector atom is excited, i.e.,

$$
t_{\text {arrival }}=\frac{\int t\left|c_{3}(t)\right|^{2} \mathrm{~d} t}{\int\left|c_{3}(t)\right|^{2} \mathrm{~d} t}
$$

(In evaluating the integrals in this equation, we use only the term in the series of equation (27) that "turns on" at $t=1 / 2$, and assume that the decay rates and distances are such that the effect of multiple scattering is negligible. These decay rates are very much faster than those displayed in the figures; the rates in the figures were chosen so that the the atomic excitation dynamics and the causal delays occurred on the same time scale, and could be illustrated on the same graphs.) The delay imposed by the medium is then just the difference in the arrival times calculated with and without a scattering atom present,

$$
\Delta t_{c_{3}}=\frac{\int t\left|c_{3}(t)\right|^{2} \mathrm{~d} t}{\int\left|c_{3}(t)\right|^{2} \mathrm{~d} t}-\frac{\int t\left|c_{3}^{0}(t)\right|^{2} \mathrm{~d} t}{\int\left|c_{3}^{0}(t)\right|^{2} \mathrm{~d} t} .
$$

To explore the effect of weak scattering we rewrite $c_{3}(t)$ in the form of equation (411), and assume that $f \ll 1$. Our quantum mechanical delay becomes, to first order in $f$,

$$
\begin{aligned}
\Delta t_{c_{3}} & =\frac{\int t\left|c_{3}^{0}(t)+f c_{3}^{\mathrm{s}}(t)\right|^{2} \mathrm{~d} t}{\int\left|c_{3}^{0}(t)+f c_{3}^{\mathrm{s}}(t)\right|^{2} \mathrm{~d} t}-\frac{\int t\left|c_{3}^{0}(t)\right|^{2} \mathrm{~d} t}{\int\left|c_{3}^{0}(t)\right|^{2} \mathrm{~d} t} \\
& \left.\simeq 2 f\left[\frac{\int t \operatorname{Re}\left[c_{3}^{0}(t) c_{3}^{\mathrm{s}}(t)^{*}\right] \mathrm{d} t}{\int\left|c_{3}^{0}(t)\right|^{2} \mathrm{~d} t}-\frac{\int t\left|c_{3}^{0}(t)\right|^{2} \mathrm{~d} t \int \operatorname{Re}\left[c_{3}^{0}(t) c_{3}^{\mathrm{s}}(t)^{*}\right] \mathrm{d} t}{\left(\int\left|c_{3}^{0}(t)\right|^{2} \mathrm{~d} t\right)^{2}}\right]\right)
\end{aligned}
$$

It is straightforward to evaluate the integrals in equation (44) using the expressions for $c_{3}^{0}(t)$ and $c_{3}^{\mathrm{s}}(t)$ from equations (30) and (31). After taking the limit $\gamma_{3} \rightarrow \infty$ and then letting $\gamma_{1} \rightarrow 0$ we arrive at the following expression for the quantum time delay:

$$
\Delta t_{c_{3}}=4 f \gamma_{2} \frac{\left(4 \delta^{2}-\gamma_{2}^{2}\right)}{\left(4 \delta^{2}+\gamma_{2}^{2}\right)^{2}} \text {. }
$$

Comparing this to equation (12) shows that "temporal-center-of-gravity" delay time for this specific pulse is identical in functional form to the classical group delay. The magnitude of the "temporal-center-of-gravity" delay is, however, twice that given by the group delay. (The "temporal-center-of-gravity" delay for classical pulses with the form given in equation (37) is also twice as large as the group delay.)

The delay in the arrival time that we have defined is the result of the reshaping of the "pulse" of excitation of the detector atom. The effect of scattering in the region of anomalous dispersion is to reduce preferentially the probability that the detector atom will be excited at large times; a short spike of probability at early times remains, shifting the "center-of-gravity" of the excitation to earlier times. Despite the appearance of negative delays, no excitation of the detector atom occurs at times $t<0.5 \mathrm{~L} / \mathrm{c}$ for any values of the parameters in our model. Although we have not demonstrated it explicitly in this work, we are confident that delays of quasimonochromatic quantum pulses can be explained in the same manner.

Because $\mathcal{E}_{\text {cl. }}, \mathcal{E}_{\text {q.m. }}$, and $c_{3}(t)$ all have the same functional form (in the large $\gamma_{3}$ limit) it is easy to see that equivalent delays can be derived from the classical field using equation (3), or from the quantum field using the analogous equation

$$
\Delta t_{\left\langle\mathcal{E}^{2}\right\rangle}=\left(\frac{\int t\left\langle: \hat{\mathcal{E}}(z=3 L / 4)^{2}:\right\rangle \mathrm{d} t}{\int\left\langle: \hat{\mathcal{E}}(z=3 L / 4)^{2}:\right\rangle \mathrm{d} t}\right)_{\text {with scatterer }}-\left(\frac{\int t\left\langle: \hat{\mathcal{E}}(z=3 L / 4)^{2}:\right\rangle \mathrm{d} t}{\int\left\langle: \hat{\mathcal{E}}(z=3 L / 4)^{2}:\right\rangle \mathrm{d} t}\right)_{\text {no scatterer }}
$$




\section{Conclusion}

We have considered simple classical and quantum models of propagation of light through dispersive media, and we have identified quantum mechanical quantities that have exact analogs in the classical scattered field. Scattering induces delays in the quantum model which are identical to the delays of a classical field. Because we use standard field-theoretic techniques of quantum optics to account for the creation and absorption of photons in our model, we have a well-defined initial condition in which all of the energy is localized at the position of the initially excited atom. This enables us to demonstrate that no effects propagate faster than the vacuum speed of light $c$, in spite of the appearance of negative delays that seemingly correspond to superluminal group velocities. In a companion paper we apply similar techniques to study delays of photons in media exhibiting electromagnetically induced transparency 28 .

\section{Appendix A. Solution using Laplace Transforms}

Taking the Laplace transform of the coupled differential equations (22)-(23) gives the coupled algebraic equations

$$
\begin{aligned}
& \mathrm{i}\left(s \tilde{c}_{1}(s)-1\right)=\sum_{m} g_{1 m} \tilde{b}_{m}(s), \\
& \mathrm{i} s \tilde{c}_{2}(s)=-\delta \tilde{c}_{2}(s)+\sum_{m} g_{2 m} \tilde{b}_{m}(s), \\
& \operatorname{is} \tilde{c}_{3}(s)=\sum_{m} g_{3 m} \tilde{b}_{m}(s), \\
& \operatorname{is} \tilde{b}_{m}(s)=m \Delta_{c} \tilde{b}_{m}(s)+\sum_{j} \tilde{c}_{j}(s) g_{j m}^{*} .
\end{aligned}
$$

The idea behind our solution is straightforward: solve these algebraic equations for the quantities $\tilde{c}_{j}(s)$ and $\tilde{b}_{m}(s)$ and then perform an inverse Laplace transform to recover the time dependence of $c_{j}(t)$ and $b_{m}(t)$. The details of carrying out such calculations are quite involved, and were completed with the aid of a computer algebra system. ${ }^{+}$In this appendix we outline our approach and present some of our intermediate results.

We begin by solving equation A.4 for $\tilde{b}_{m}(s)$, and substitute the result in the first three equations, giving

$$
\begin{aligned}
& s \tilde{c}_{1}-1=-\mathrm{i} \Delta_{\mathrm{c}}\left(f_{11} \tilde{c}_{1}+f_{12} \tilde{c}_{2}+f_{13} \tilde{c}_{3}\right), \\
& (s-\mathrm{i} \delta) \tilde{c}_{2}=-\mathrm{i} \Delta_{\mathrm{c}}\left(f_{21} \tilde{c}_{1}+f_{22} \tilde{c}_{2}+f_{23} \tilde{c}_{3}\right), \\
& s \tilde{c}_{3}=-\mathrm{i} \Delta_{\mathrm{c}}\left(f_{31} \tilde{c}_{1}+f_{32} \tilde{c}_{2}+f_{33} \tilde{c}_{3}\right),
\end{aligned}
$$

in which we have defined the dimensionless sums

$$
f_{l n}=\frac{1}{\Delta_{\mathrm{c}}^{2}} \sum_{m} \frac{g_{l m} g_{n m}^{*}}{\frac{\mathrm{i} s}{\Delta_{\mathrm{c}}}-m}
$$

In the limit in which the atomic resonance frequencies are very much greater than the fundamental frequency of the cavity, i.e., $\omega_{j}^{(\text {at })} \gg \Delta_{c}$, these sums may be

\footnotetext{
+ Mathematica notebooks used to perform the calculations are available from the authors.
} 
approximated by extending the range for $m$ from $-\infty$ to $+\infty$, in which case the sums have relatively simple representations in terms of trigonometric functions. An explicit example of one of these sums (for atoms at positions $z_{1}=L / 4, z_{2}=L / 2$, and $\left.z_{3}=3 L / 4\right)$ is

$$
\begin{aligned}
f_{11} & \simeq \frac{\gamma_{1}}{8 \Delta_{\mathrm{c}}}\left\{\cot \left[\frac{\pi}{4}\left(\frac{\mathrm{i} s}{\Delta_{\mathrm{c}}}-1\right)\right]+2 \cot \left[\frac{\pi}{4}\left(\frac{\mathrm{i} s}{\Delta_{\mathrm{c}}}-2\right)\right]+\cot \left[\frac{\pi}{4}\left(\frac{\mathrm{i} s}{\Delta_{\mathrm{c}}}-3\right)\right]\right\} \\
& =-\mathrm{i}\left(\frac{\gamma_{1}}{4 \Delta_{\mathrm{c}}}\right) \frac{\sinh \frac{3 \pi s}{4 \Delta_{\mathrm{c}}}}{\cosh \frac{\pi s}{4 \Delta_{\mathrm{c}}} \cosh \frac{\pi s}{2 \Delta_{\mathrm{c}}}} .
\end{aligned}
$$

After solving equations A.5 - A.7) for the quantities $\tilde{c}_{j}(s)$ in terms of the sums $f_{j m}$, we rewrite the hyperbolic trigonometric functions resulting from the sums in terms of exponential functions; we also let $c / L=1$ at this point in the calculation. We then expand the resulting expressions in powers of $\exp (-s / 4)$, and the time dependence of the system is recovered by a term-by-term inverse Laplace transform of the expansion. The step function turn-on of the resulting time dependence arises because of the factors $\exp (-n s / 4)$ in the expansion. The lowest order terms in our expansions of the Laplace transforms are given here:

$$
\begin{aligned}
& \tilde{c}_{1}(s)=\frac{2}{2 s+\gamma_{1}}+\frac{4 \exp (-s / 2) \gamma_{1}\left(s+\gamma_{2}-\mathrm{i} \delta\right)}{\left(2 s+\gamma_{1}\right)^{2}\left(2 s+\gamma_{2}-\mathrm{i} 2 \delta\right)}+\cdots \\
& \tilde{c}_{2}(s)=-\frac{2 \exp (-s / 4) \sqrt{\gamma_{1} \gamma_{2}}}{\left(2 s+\gamma_{1}\right)\left(2 s+\gamma_{2}-\mathrm{i} 2 \delta\right)}+\cdots \\
& \tilde{c}_{3}(s)=-\frac{4 \exp (-s / 2) \sqrt{\gamma_{1} \gamma_{3}}(s-\mathrm{i} \delta)}{\left(2 s+\gamma_{1}\right)\left(2 s+\gamma_{3}\right)\left(2 s+\gamma_{2}-\mathrm{i} 2 \delta\right)}+\cdots \\
& \tilde{b}_{m}(s)=\frac{2 \mathrm{i}}{(s+\mathrm{i} m \pi)\left(2 s+\gamma_{1}\right)}\left[\frac{\exp (-s / 4) \sqrt{\gamma_{1} \gamma_{2}} g_{2 m}}{\left(2 s+\gamma_{2}-\mathrm{i} 2 \delta\right)}-g_{1 m}\right] .
\end{aligned}
$$

The inverse Laplace transform of these expressions gives equations (25)- 281).

To calculate $\mathcal{E}_{\text {q.m. }}$. of equation (36) we repeat the process with the detector atom at $z=3 L / 4$ removed, yielding

$$
\begin{aligned}
& \tilde{c}_{1}(s)=\frac{2}{2 s+\gamma_{1}}+\cdots \\
& \tilde{c}_{2}(s)=-\frac{2 \exp (-s / 4) \sqrt{\gamma_{1} \gamma_{2}}}{\left(2 s+\gamma_{1}\right)\left(2 s+\gamma_{2}-\mathrm{i} 2 \delta\right)}+\cdots \\
& \tilde{b}_{m}(s)=\frac{2}{\left(\mathrm{i} s-m \Delta_{\mathrm{c}}\right)\left(2 s+\gamma_{1}\right)}\left(g_{1 m}^{*}-g_{2 m}^{*} \frac{\sqrt{\gamma_{1} \gamma_{2}} \exp (-s / 4)}{\left(2 s+\gamma_{2}-\mathrm{i} 2 \delta\right)}\right)
\end{aligned}
$$

Using the same techniques as above, the Laplace transform of $\mathcal{E}_{\text {q.m. }}$. can be written as

$$
\begin{aligned}
\tilde{\mathcal{E}}_{\mathrm{q} . \mathrm{m} .} & =\sqrt{\frac{2 \hbar \omega_{1}^{(\mathrm{at})}}{\epsilon_{0} V} \sum_{m}} \tilde{b}_{m}(s) \sin \left(\frac{m 3 \pi}{4}\right) \\
& \rightarrow-\mathrm{i} \sqrt{\frac{2 \hbar \omega_{1}^{(\mathrm{at})} \gamma_{1}}{\pi \Delta_{\mathrm{c}} \epsilon_{0} V}} \frac{\exp (-s / 2)}{\left(2 s+\gamma_{1}\right)}\left[1-\frac{\gamma_{2}}{\left(2 s+\gamma_{2}-\mathrm{i} 2 \delta\right)}\right] .
\end{aligned}
$$

The inverse Laplace transform of equation (A.17) gives equation (36). 
Classical Wave Delays in Quantum Scattering

\section{Acknowledgments}

The authors thank James Supplee for helpful discussions and careful reading of the manuscript. One of us (T.P.) acknowledges support from National Science Foundation Research Experiences for Undergraduates Program (Grant Number PHY-0097424).

\section{References}

[1] L. Brillouin, Wave Propagation and Group Velocity (Academic, New York, 1960).

[2] L. V. Hau, S. E. Harris, Z. Dutton, and C. H. Behroozi, Nature 397, 594 (1999).

[3] M. M. Kash, V. A. Sautenkov, A. S. Zibrov, L. Hollberg, G. R. Welch, M. D. Lukin, Y. Rostovtsev, E. S. Fry, and M. O. Scully, Phys. Rev. Lett. 82, 5229 (1999).

[4] A. M. Steinberg, P. G. Kwiat, and R. Y. Chiao, Phys. Rev. Lett. 71, 708 (1993).

[5] L. J. Wang, A. Kuzmich, and A. Dogariu, Nature 406, 277 (2000).

[6] R. Chiao and A. Steinberg, in Progress in Optics, edited by E. Wolf (Elsevier, 1997), vol. 37, pp. 345-405.

[7] R. P. Feynman, R. B. Leighton, and M. Sands, The Feynman Lectures on Physics (AddisonWesley, Reading, MA, 1963), vol. I, chap. 31 and 32.

[8] B. A. Sherwood, Am. J. Phys. 64, 840 (1996).

[9] P. W. Milonni, Am. J. Phys. 64, 842 (1996).

[10] D. J. Griffiths, Introduction to Electrodynamics (Prentice Hall, Upper Saddle River, NJ, 1999), chap. 9, 3rd ed.

[11] D. F. Taylor, Undergraduate Honors Thesis, Bucknell University (2001).

[12] M. Ligare and D. F. Taylor (2001), paper presented at the Eighth Rochester Conference on Coherence and Quantum Optics, URL http://www.eg.bucknell.edu/physics/ligare.html/

[13] M. Ligare and R. Oliveri, Am. J. Phys. 70, 58 (2002).

[14] V. Bužek, G. Drobný, M. G. Kim, M. Havukainen, and P. L. Knight, Phys. Rev. A 60, 582 (1999).

[15] G. Drobný, M. Havukainen, and V. Bužek, J. Mod. Optics 47, 851 (2000).

[16] R. L. Smith, Am. J. Phys. 38, 978 (1970).

[17] S. C. Bloch, Am. J. Phys. 45, 538 (1977).

[18] P. Meystre and M. Sargent, Elements of Quantum Optics (Springer, Berlin, 1999), 3rd ed.

[19] M. Sargent, M. O. Scully, and W. E. Lamb, Laser Physics (Addison-Wesley, Reading, MA, 1974).

[20] R. Loudon, The Quantum Theory of Light (Oxford U. P., Oxford, 1983), 2nd ed.

[21] G. C. Stey and R. W. Gibberd, Physica 60, 1 (1972).

[22] P. W. Milonni and P. L. Knight, Phys. Rev. A 10, 1096 (1974).

[23] P. W. Milonni and P. L. Knight, Phys. Rev. A 11, 1090 (1975).

[24] H. Gießen, J. D. Berger, G. Mohs, and P. Meystre, Phys. Rev. A 53, 2816 (1996).

[25] M. O. Scully and M. S. Zubairy, Quantum Optics (Cambridge University Press, Cambridge, 1997).

[26] M. Orszag, Quantum Optics (Springer-Verlag, Berlin, 2000), chap. 15, and references therein.

[27] V. Peřinová, A. Lukš, and J. Peřina, Phase in Optics (World Scientific, Singapore, 1982), chap. 4, and references therein.

[28] T. Purdy and M. Ligare, submitted to J. Opt. B: Quantum Semiclass. Opt., quant-ph/0204173 